Research Article

\title{
Light-Harvesting Metal-Organic Frameworks (MOFs) La-PTC for Photocatalytic Dyes Degradation
}

\author{
Agustino Zulys, Adawiah Adawiah*), Jarnuzi Gunlazuardi, Muhammad Derry Luthfi Yudhi \\ Department of Chemistry, Faculty of Mathematics and Natural Sciences, University of Indonesia, \\ Jl. Lingkar Kampus Raya, Pondok Cina, Beji, Depok, Jawa Barat 16424, Indonesia.
}

Received: $8^{\text {th }}$ February 2021; Revised: 20th March 2021; Accepted: 21 st March 2021

Available online: 22th March 2021; Published regularly: March 2021

\section{Abstract}

A novel porous metal organic framework, La-PTC was synthesized by solvothermal method using a perylene3,4,9,10-tetracarboxylate ligand and lanthanum metal ion. The FTIR analysis showed that La-PTC has a different structure with PTCDA and $\mathrm{Na}_{4}$ PTC. The La-PTC MOF has high crystallinity, bandgap energy of $2.21 \mathrm{eV}$ with a maximum absorption area at $561 \mathrm{~nm}$. A rod shape structure of La-PTC has been obtained with the surface area of $22.2364 \mathrm{~m}^{2} \cdot \mathrm{g}^{-1}$ and classified into mesoporous material. The La-PTC was relative stable up to $376.93{ }^{\circ} \mathrm{C}$. The LaPTC can degrade $64.76 \%$ of MO within ca. 240 min under visible light irradiation with the amount of $30 \mathrm{mg}$ LaPTC. The addition of $\mathrm{H}_{2} \mathrm{O}_{2}$ improved the photocatalytic activity of La-PTC with degradation efficiency of $67.02 \%$, $70.00 \%$, and $99.60 \%$ for $\mathrm{MB}, \mathrm{RhB}$, and MO, respectively. This study presents the fabrication of the light-harvesting metal organic framework, La-PTC and its potential in dyes degradation.

Copyright (C) 2021 by Authors, Published by BCREC Group. This is an open access article under the CC BY-SA License (https://creativecommons.org/licenses/by-sa/4.0).

Keywords: Metal-organic frameworks; lanthanum; perylene; dyes degradation; photocatalytic

How to Cite: A. Zulys, A. Adawiah, J. Gunlazuardi, M.D.L. Yudhi (2021). Light-Harvesting Metal-Organic Frameworks (MOFs) La-PTC for Photocatalytic Dyes Degradation. Bulletin of Chemical Reaction Engineering \& Catalysis, 16(1), 170-178 (doi:10.9767/bcrec.16.1.10309.170-178)

Permalink/DOI: https://doi.org/10.9767/bcrec.16.1.10309.170-178

\section{Introduction}

Photocatalysis technology has appealed to interest for inexpensive material and environmentally friendly nature in carrying out dyecontaining wastewater. Nowadays, several semiconductor materials are applied as photocatalysts for the efficient elimination of dyes in wastewater. Currently, semiconductors, such as: $\mathrm{TiO}_{2}$ [1], $\mathrm{ZnO}_{2}-\mathrm{WO}_{3}$ [2], and other metal oxides, are exploited as photocatalysts for photocatalytic dyes degradation purposes. Nevertheless, in practice, their application is greatly limited due

\footnotetext{
* Corresponding Author.

Email: adawiah@ui.ac.id (A. Adawiah);

Telp: +62-21-7270027, Fax: +62-21-7270277
}

to the application under ultraviolet light irradiation [3], limited adsorption capacity and difficult separation process. Therefore, the fabrication of a material which can cope with this limitation is being important.

Metal-organic frameworks (MOFs), a class of porous crystalline material, which are designed by metal ion or cluster metal ion coordinated organic linkers [4], have enhancing interest due to their unique properties and tunable structure. The MOFs have been applied in many applications including adsorption, separation and photocatalysis [5]. The MOFs are potential materials for the removal of dyes contaminant in wastewater [6] due to the characteristics which applicable in water treatment, such as: large surface area, abundant active sites, high stabil- 
ity in water, acid, base, tunable structure, and pore size [7].

Lanthanide metal ions with high versatile coordination modes are widely adopted for the fabrication of MOFs. Xia and co-workers in 2017 reported that Ln-MOFs was synthesized from an anthracene-coordinated organic linker with $\mathrm{Ce}, \mathrm{Tb}$ and $\mathrm{Dy}$ metal ion exhibit highly rhodamine $\mathrm{B}(\mathrm{RhB})$ degradation under visible light irradiation in the presence of $\mathrm{H}_{2} \mathrm{O}_{2}$ [8]. Other MOFs such as lanthanum-based metalorganic frameworks (La-MOFs) linked by aromatic dicarboxylic acid ligand [9] and lanthanide-based metal-organic frameworks (LnMOFs) with chalcone dicarboxylic acid ligand [10] also has been reported for the degradation of RhB. Nevertheless, there have been limited studies concerned with anionic dye degradation by MOFs, particularly La-MOFs. Therefore, this research report the visible light responsive lanthanum-based MOF which has been fabricated using perylene-3,4,9,10-tetracarboxylate (PTC) as the ligand. To the best of our knowledge, PTC has never been used as the ligand for the La-MOFs. The lanthanum-based metal-organic frameworks linked by perylene3,4,9,10-tetracarboxylate (La-PTC) was synthesized using a solvothermal method with $\mathrm{N}, \mathrm{N}$ dimethylformamide (DMF) as the solvent. The synthesized La-PTC then be used as the photocatalyst for cationic (methylene blue (MB), Rhodamin B (RhB)) and anionic (methyl orange (MO)) dye degradation under visible light irradiation.

\section{Materials and Methods}

\subsection{Chemicals and Instrumentation}

All chemicals were analytical grade and were used without further purification. Lanthanum nitrate hexahydrate $\left(\mathrm{La}\left(\mathrm{NO}_{3}\right)_{3} \cdot 6 \mathrm{H}_{2} \mathrm{O}\right)$, sodium hydroxide $(\mathrm{NaOH})$, ethanol, hydrogen peroxide $\left(\mathrm{H}_{2} \mathrm{O}_{2}\right)$, rhodamine $\mathrm{B}(\mathrm{RhB})$, methylene blue (MB), methyl orange (MO), tertbutyl alcohol (TBA), and methanol were supplied by Merck. Perylene-3,4,9,10tetracarboxylic anhydride (PTCDA) was supplied by Sigma Aldrich Chemical Co. Characterization of La-PTC was carried out using FTIR with $\mathrm{KBr}$ pellet for the background measurement on IR Prestige-21 Shimadzu (4000$400 \mathrm{~cm}^{-1}$ ). The PXRD Shimadzu XRD 7000 Maxima-X $(\mathrm{Cu}-\mathrm{K} \alpha$ radiation $(\lambda=1.5418 \AA)$ was used with the scan rate of $2^{\circ} \mathrm{min}^{-1}$ at room temperature on the $2 \theta$ angle of $2^{\circ}-80^{\circ}$. The UVDRS spectra were analyzed by using UV-Vis Spectrophotometer Agilent Carry 60 with Ba$\mathrm{SO}_{4}$ as reference at wavelength of 200-800 nm.
The scanning electron microscopy (SEM) images were obtained on a FEI Quanta 650. The specific surface area was measured using the Brunauer-Emmett-Teller (BET) method on a Micromeritics TriStar II Surface Area and Porosity. Thermogravimetric analysis (TGA) was measured by TGA Q50 V20.13 Build 39 at the rate of heating was $10{ }^{\circ} \mathrm{C} \mathrm{min}{ }^{-1}$ under air atmosphere.

\subsection{Preparation of Sodium Perylene-3,4,9,10-} tetracarboxylate $\left(\mathrm{Na}_{4} \mathrm{PTC}\right)$

PTCDA (0.5 g, $1.27 \mathrm{mmol})$ were dissolved in distilled water $(50 \mathrm{~mL})$ on a beaker glass. $\mathrm{NaOH}(0.356 \mathrm{~g}, 8.9 \mathrm{mmol})$ were added to the mixture while stirring vigorously at $300 \mathrm{rpm}$ for 1 hour. The greenish yellow solution was obtained and filtered. Then, excess ethanol was added to obtain a yellow sodium perylene3,4,9,10-tetracarboxylate ( $\left.\mathrm{Na}_{4} \mathrm{PTC}\right)$ precipitate. The yellow bulk powder of $\mathrm{Na}_{4} \mathrm{PTC}$ were collected by filtration, washed with ethanol until a $\mathrm{pH}$ of 7 is reached and dried at room temperature overnight.

\subsection{Preparation of La-PTC}

The La-PTC was synthesized by using a solvothermal method. Typically, $\mathrm{La}\left(\mathrm{NO}_{3}\right)_{3} \cdot 6 \mathrm{H}_{2} \mathrm{O}$ (866 $\mathrm{mg}, 2 \mathrm{mmol}$ ) as the metal source and $\mathrm{Na}_{4}$ PTC (508 mg, $1 \mathrm{mmol}$ ) as the organic ligand were dissolved in a mixture of DMF and water (5:1 volume ratio, respectively) and magnetically stirred at $300 \mathrm{rpm}$ for 60 minutes. Then, the solution was transferred to the Teflon autoclave. The Teflon autoclave was placed in the oven for 24 hours at $170{ }^{\circ} \mathrm{C}$ and allowed to cool to room temperature overnight. The orange crystal La-PTC obtained was collected by filtration and washed with DMF and distilled water to eliminate the impurities. The bulk compound of La-PTC then was dried at $70{ }^{\circ} \mathrm{C}$ overnight.

2.4 Photocatalytic Degradation of Dyes Experiment

Photocatalytic activity of La-PTC for $\mathrm{MB} / \mathrm{RhB} / \mathrm{MO}$ degradation was investigated with the 250 watts mercury lamp as the visible light source. La-PTC (30 mg) was dispersed in $50 \mathrm{~mL}$ of $\mathrm{MB} / \mathrm{RhB} / \mathrm{MO}$ solution $(10 \mathrm{mg} / \mathrm{L})$. The suspension was magnetically stirred at 300 $\mathrm{rpm}$ and irradiated by the mercury lamp for 240 minutes. In addition, a fixed amount of $\mathrm{H}_{2} \mathrm{O}_{2} 30 \%, \mathrm{CH}_{3} \mathrm{OH}$ and tert-butyl alcohol (TBA) were added into the system in order to determine the role of the electron-hole scavenger. 
Then, $2.0 \mathrm{~mL}$ of the suspension was taken out at different time intervals and centrifugation. The concentration of $\mathrm{MB}, \mathrm{RhB}$, and $\mathrm{MO}$ were determined using a UV-Vis spectrophotometer at $665 \mathrm{~nm}, 555 \mathrm{~nm}$ and $465 \mathrm{~nm}$, respectively. The several control experiments were performed under dark conditions and without LaPTC at the same condition. The degradation efficiency of $\mathrm{MB} / \mathrm{RhB} / \mathrm{MO}$ was calculated by using the equation (1):

$$
D E(\%)=\frac{C_{0}-C_{t}}{C_{0}} \times 100 \%
$$

$C_{0}$ and $C_{\mathrm{t}}$ assert the initial and final concentration of $\mathrm{MB} / \mathrm{RhB} / \mathrm{MO}$ solution, respectively.

\section{Results and Discussion}

A yellow bulk compound of $\mathrm{Na}_{4} \mathrm{PTC}$ was obtained from the base hydrolysis of PTCDA. The functional groups of $\mathrm{Na}_{4} \mathrm{PTC}$ were investigated by FTIR in the range $4000-400 \mathrm{~cm}^{-1}$ (Figure 1).

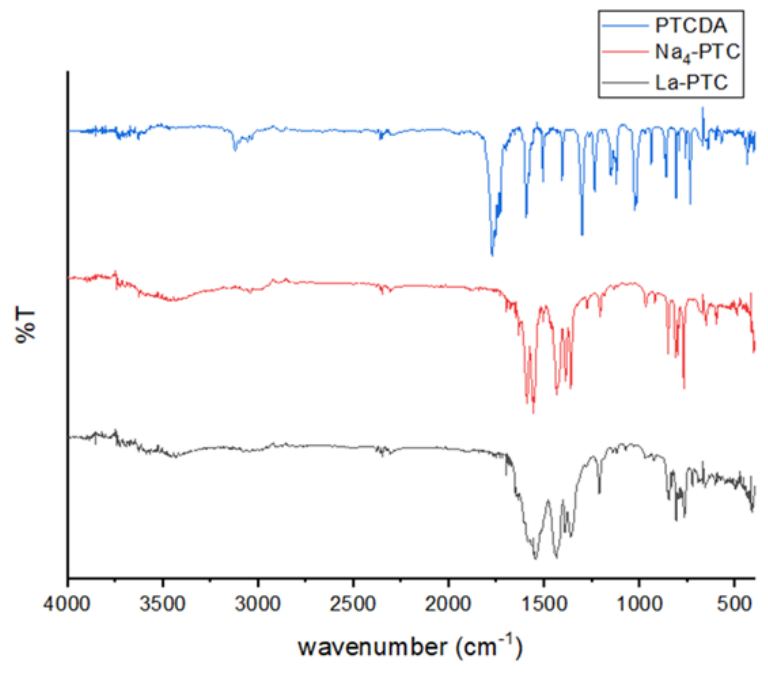

Figure 1. FTIR spectrum of PTCDA, $\mathrm{Na}_{4} \mathrm{PTC}$ and La-PTC.
The strong peak at ca. $1772 \mathrm{~cm}^{-1}$ and 1730 $\mathrm{cm}^{-1}$ are related to the asymmetric and symmetric $\mathrm{C}=\mathrm{O}$ stretching vibration of the cyclic anhydride group in PTCDA. The peak at ca. $1234 \mathrm{~cm}^{-1}$ due to vibration of $\mathrm{C}-\mathrm{CO}-\mathrm{O}-\mathrm{CO}-\mathrm{C}$ cyclic anhydride group in PTCDA does not appear in the $\mathrm{Na}_{4} \mathrm{PTC}$ spectrum. In addition, the new peak at ca. $1633 \mathrm{~cm}^{-1}$ and $1423 \mathrm{~cm}^{-1}$ formed which are identified as asymmetric and symmetric stretching vibration $(-\mathrm{COO})$ group of the carboxylate ion functional group. This indicated that PTCDA was converted to carboxylate ion.

The FTIR spectrum (Figure 1) also showed that La-PTC has different band absorption with $\mathrm{Na}_{4} \mathrm{PTC}$. The sharp peaks at ca. 1633 $\mathrm{cm}^{-1}$ and $1423 \mathrm{~cm}^{-1}$ in $\mathrm{Na}_{4} \mathrm{PTC}$ were shifted to $1538 \mathrm{~cm}^{-1}$ and $1435 \mathrm{~cm}^{-1}$, respectively. This indicated that La-PTC was formed and all the carboxylate ion group from $\mathrm{Na}_{4} \mathrm{PTC}$ were completely deprotonated with $\mathrm{La}^{3+}$ ion and $-\mathrm{COO}$ functional group in PTCDA was coordinated with $\mathrm{La}^{3+}$.

The phase of La-PTC was examined by XRD. The diffractogram showed sharp peaks at ca. $2 \theta$ angle $=6.31^{\circ}, 12.64^{\circ}, 16.6^{\circ}, 28.7^{\circ}, 33.3^{\circ}$, $44.6^{\circ}, 47.9^{\circ}, 50.9^{\circ}, 59.6^{\circ}, 62.3^{\circ}, 70^{\circ}, 77.3^{\circ}$ with the highest intensity observed at ca. $2 \theta$ angle $=$ $6.31^{\circ}, 16.6^{\circ}, 28.7^{\circ}, 33.3^{\circ}$ and $44.6^{\circ}$ (Figure 2). This indicated that La-PTC has high crystallinity.

The La-PTC optical properties were measured by a UV-Vis spectrophotometer in order to ensure the band gap energy of the La-PTC. The band gap energy was calculated using the Kubelka-Munk (eq. 2) and the Tauc plot (eq. 3) as shown below:

$$
\begin{gathered}
F(R)=\frac{(1-R)^{2}}{2 R} \\
{[\alpha h v]=A[h v-E g]^{1 / 2}}
\end{gathered}
$$

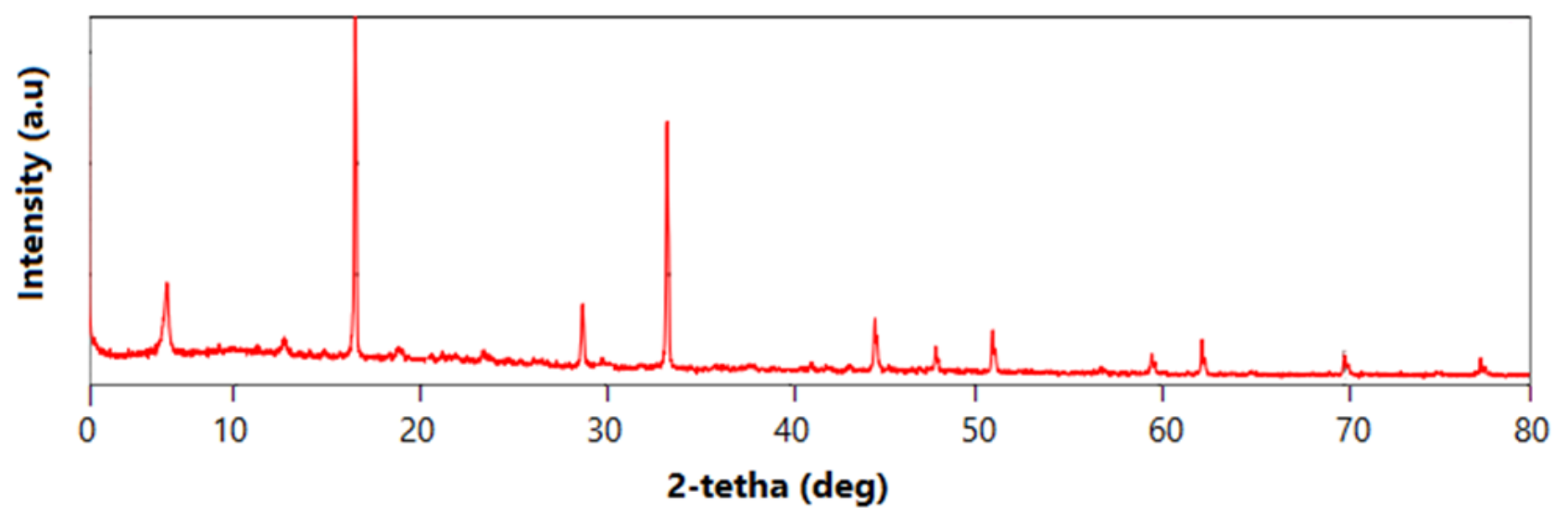

Figure 2. Diffraction pattern of La-PTC. 
where $E_{\mathrm{g}}$ is the band gap energy $(\mathrm{eV}), h$ is Planck's constant, $v$ is the frequency of light, $A$ is the absorption constant and $\alpha=F(R)$ is the absorption coefficient, $1 / 2$ is electronic

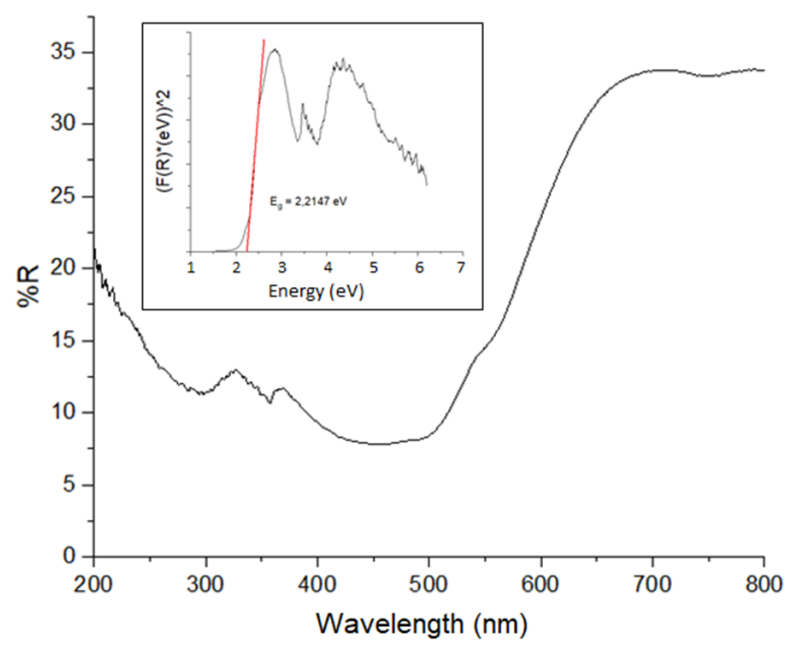

Figure 3. DRS-UV spectra and Tauc plot of La-PTC.
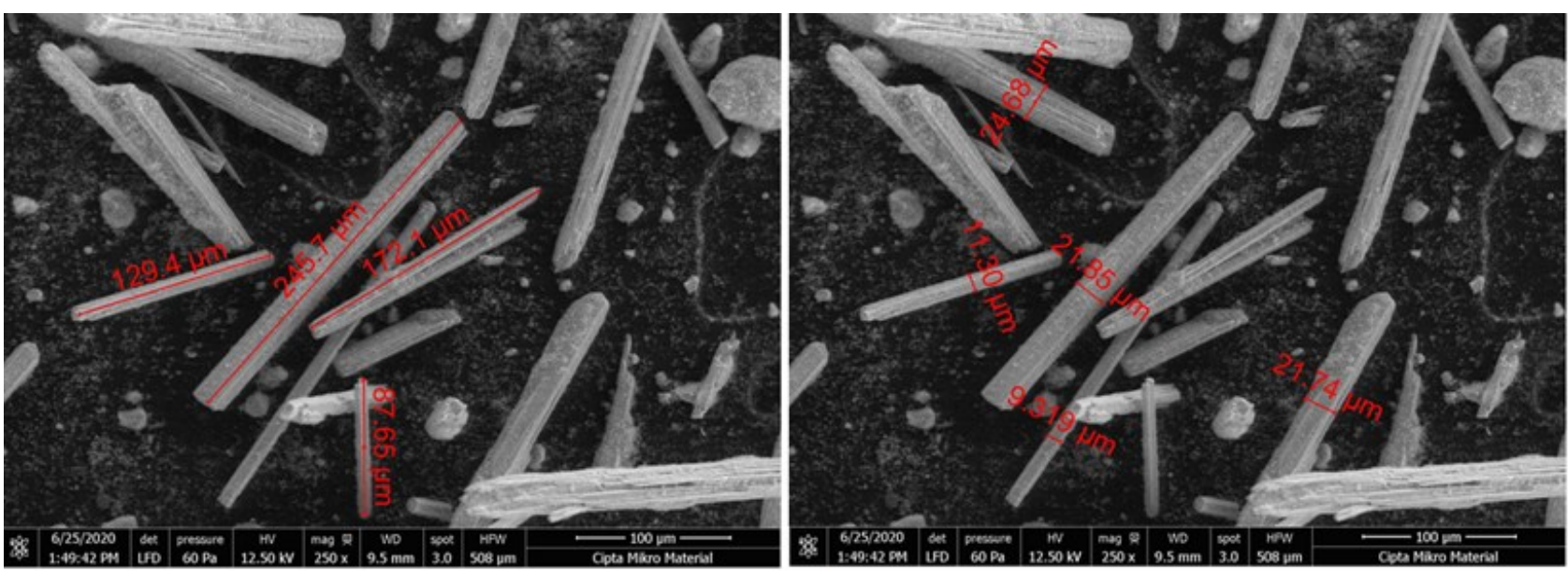

(a)

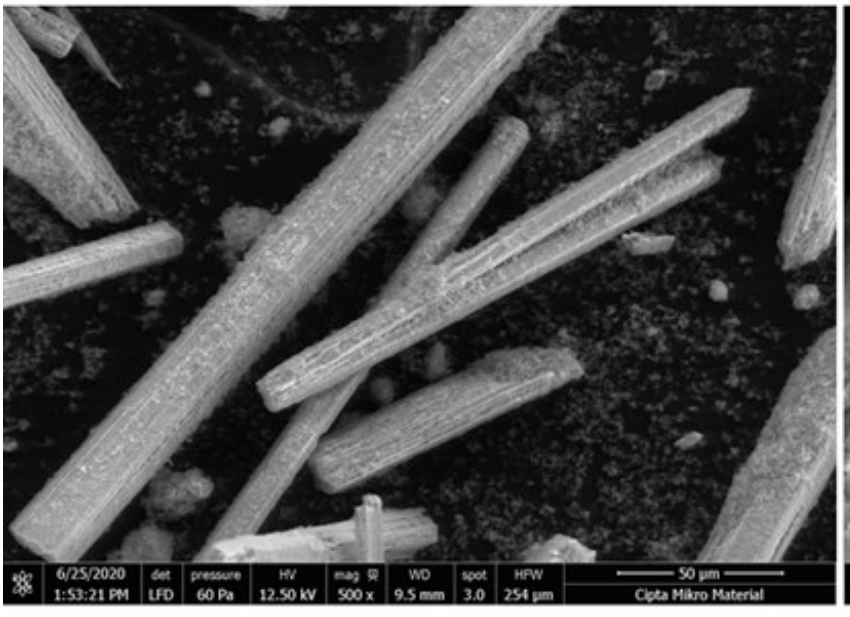

(b) transtition number for direct allowed transtition material, respectively. A plot of $[\mathrm{F}(\mathrm{R}) \mathrm{h} v]^{1 / 2}$ vs $h v$ can be achieved using the $\mathrm{y}$ value of zero. The $E_{\mathrm{g}}$ was acquired by calculated the $\mathrm{x}$-axis intercept of an extrapolated tangential line from the linearity of the curve.

As shown in Figure 3, the main optical absorption band of La-PTC was around $561 \mathrm{~nm}$, or the $E_{\text {g }}$ was estimated to be $2.21 \mathrm{eV}$ by using linear equation of $y=686.88 x-1551.3\left(R^{2}=\right.$ 0.9955). La-PTC show broad-range visible light absorption due to the presence of highly conjugated pi $(\pi)$ of perylene structure. The $E_{\mathrm{g}}$ of perylene tetracarboxylate ligand-based LaMOF was lower than 2,6-naphthalene dicarboxylic acid ligand-based La-MOF that is 3.10 $\mathrm{eV}$ [11]. The constriction band gap energy occurs due to the electronic transition effect $\pi-\pi^{*}$ on the conjugated $\pi$ bond of the perylene ligand. Perylene has more conjugated $\pi$ bonds than 2,6-naphthalene dicarboxylic acid. In the conjugated $\pi$ bonding system, there is an overlap of orbitals, which will reduce the gap ener-

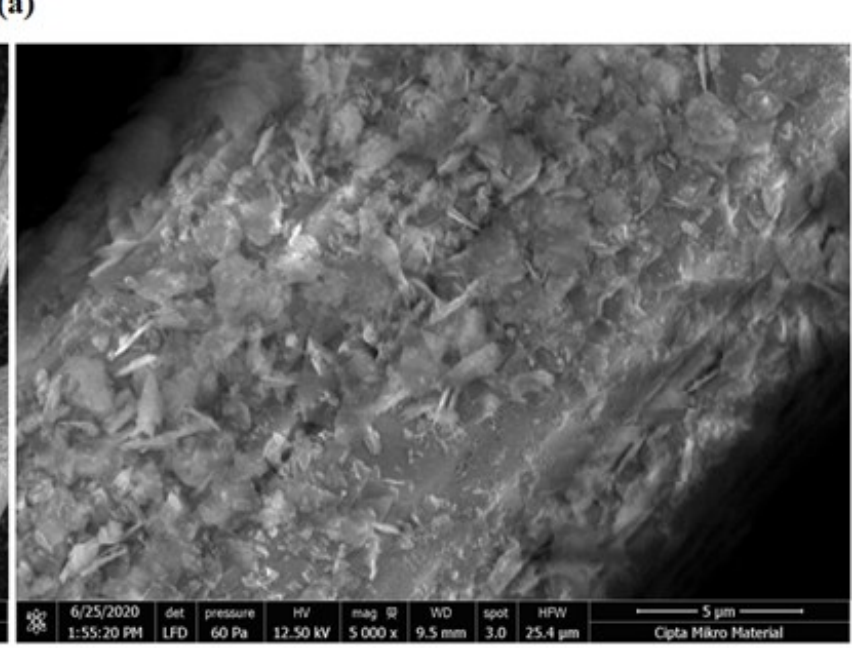

(c)

Figure 4. Morphology of La-PTC with (a) 250x, (b) 500x, and (c) 5000x magnification. 
gy between the neighboring orbitals and leads to a bathochromic shift in the absorption spectrum.

On the other hand, in the conjugated bonding system, when an electron absorbs photons at the right wavelength, the electron will be normalized to a higher energy level. Commonly, the electron transition occurs in $\Pi$ to antibonding $\Pi$ orbitals $\left(\pi-\pi^{*}\right)$ electron. A conjugated system with conjugated double bonds of less than eight members can only absorb the energy around the ultraviolet area, so they will appear colorless. With the addition of a double bond, the system will absorb photons from the wider wavelength area. For example, the naphthacene (four rings) has maximum absorption at $470 \mathrm{~nm}$, while pentacene (five rings) has a maximum absorption at $575 \mathrm{~nm}$.

Figure 4 showed that the morphology of LaPTC has a rod shape structure with various lengths and diameters. The structure of LaPTC has a good homogeneity as indicated by the crystal structure having almost the same morphology at each observation point. Meanwhile, the EDS characterization showed the elements of the La-PTC which consisted of carbon, oxygen and lanthanum with amounts of $51.8 \%, 28.3 \%$, and $19.9 \%$, respectively.

La-PTC has a type II adsorption isotherm as shown from the BET analysis in Figure 5a. The presence of an initial curve pattern indicated the occurrence of monolayer or multilayer adsorption on the material. The isotherm graph did not show a sharp increment in adsorption, so it is indicated that when the entire surface of the monolayer is covered by adsorbate, multilayer adsorption occurs. At the beginning of the curve, there was a very sharp increment. It is indicated that monolayer adsorption has oc-

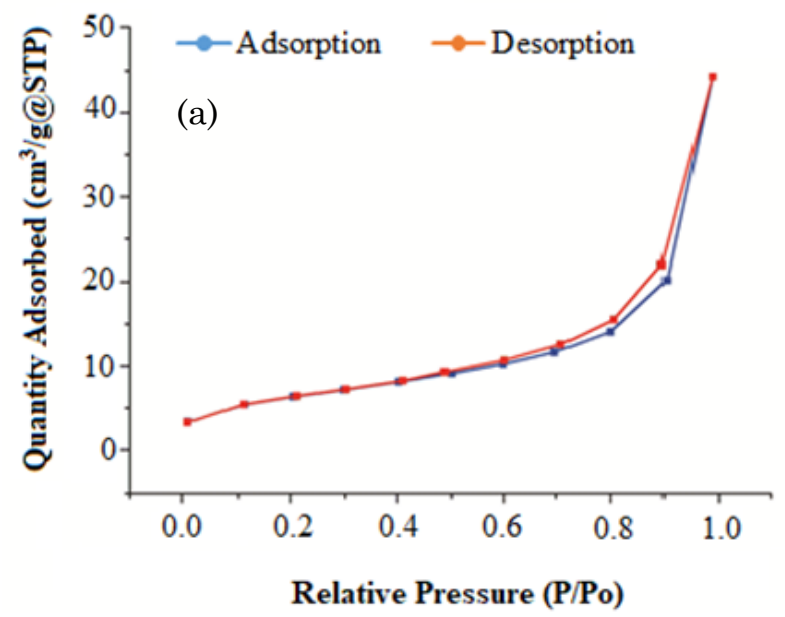

curred overall. Whereas, if there is no sharp increment at the beginning of the curve, multilayer adsorption begins when the entire surface of the monolayer is covered by adsorbate [12].

La-PTC has a surface area of 22.2364 $\mathrm{m}^{2} \cdot \mathrm{g}^{-1}$. The surface area is relatively smaller when compared to the surface area of perylene based MOF based on different metals which is $36.228 \mathrm{~m}^{2} . \mathrm{g}^{-1}$ for Dy-MOF; $51.853 \mathrm{~m}^{2} \cdot \mathrm{g}^{-1}$ for Sm-MOF [13]; and $67.779 \mathrm{~m}^{2} \cdot \mathrm{g}^{-1}$ for Ni-MOF [14]. In addition, the pore size distribution of La-PTC was found at the range of $2-25 \mathrm{~nm}$ (Figure 5b). It is assumed that La-PTC was a mesoporous material group, which has the advantage of being a photo-catalyst that can provide a short distance to reduce the recombination of photo-excited electron-hole [15].

Thermogravimetric analysis was carried out to ensure the temperature at which the material tends to stabilize and begin to decompose and what compounds are produced when the materials were decomposed. The TGA analysis exhibits the La-PTC material relatively stable at temperatures of 0 to $376.39{ }^{\circ} \mathrm{C}$. It suggested that the La-PTC material was stable at high temperatures.

The methylene blue (MB), rhodamin B (RhB) and methyl orange (MO) photocatalytic degradation were investigated in order to determine the efficiency of La-PTC as photocatalysts in $\mathrm{MB}, \mathrm{RhB}$ and $\mathrm{MO}$ degradation under visible irradiation. Several control experiments were designed to clarify the properties of photocatalytic reactions. Figure 6 showed that the adsorption-desorption experiment (MB/RhB/MO + La-PTC in the dark condition) exhibits the dyes molecules can be absorbed by La-PTC with an adsorption capacity of $8.4 \%$ for

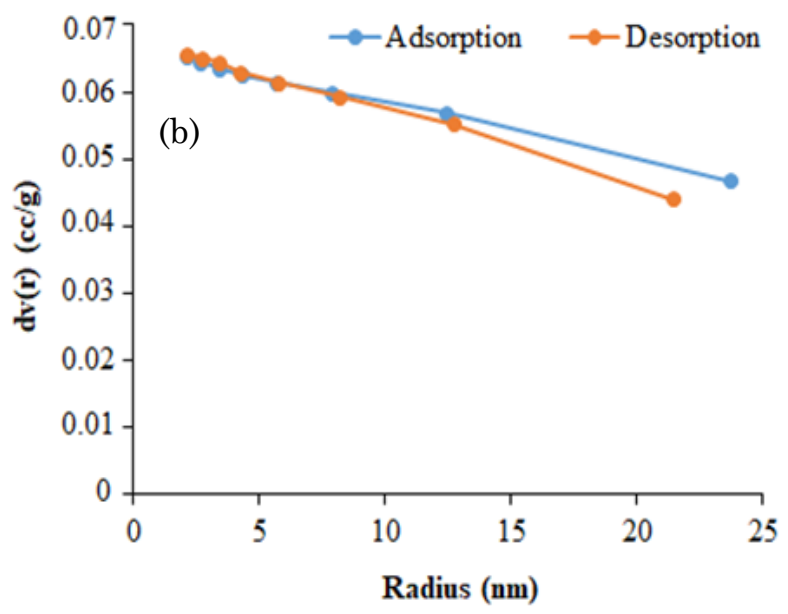

Figure 5. La-PTC (a) adsorption-desorption and (b) pore size distribution curves. 
$\mathrm{MB}, 25.55 \%$ for $\mathrm{RhB}$ and $16.71 \%$ for MO. The photolysis experiment shows the $\mathrm{MB}, \mathrm{RhB}$, and MO are slightly stable for 240 minutes of irradiation with and without addition of $\mathrm{H}_{2} \mathrm{O}_{2}$. LaPTC shows no photocatalytic activity to degrade $\mathrm{MB}$ and $\mathrm{RhB}$, but it degraded $\mathrm{MO}$ with a degradation efficiency of $64.74 \%$ for 240 minutes irradiation. It is indicated that the di-

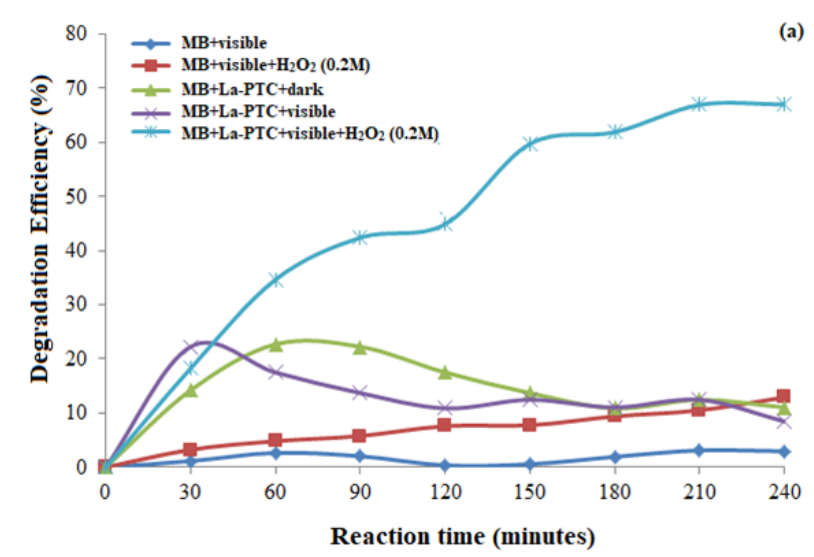

(a)

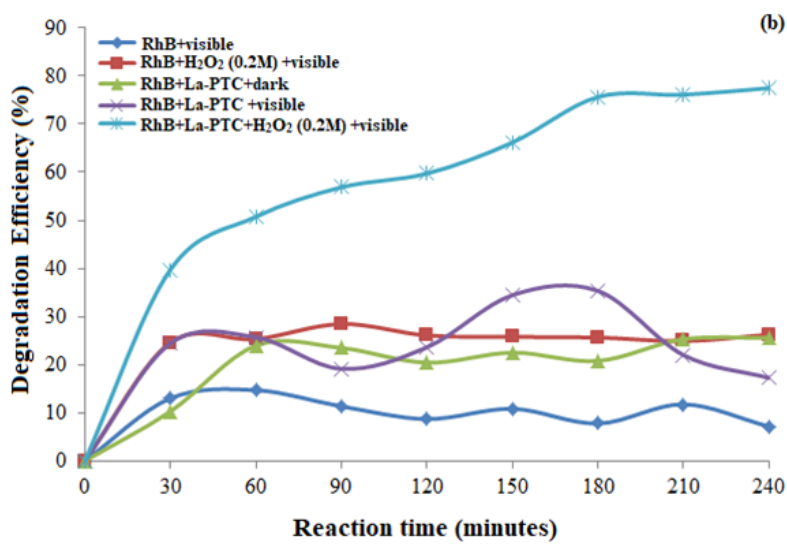

(b) rect photocatalytic degradation of $\mathrm{MB}$ and $\mathrm{RhB}$ by La-PTC was less energetic favorable.

Figure $7 \mathrm{a}$ showed the conduction band (CB) of La-PTC $(-1.60 \mathrm{~V})$ which is less negative than the LUMO of MB (-0.25 eV) and RhB (-1.00 $\mathrm{eV}$ ). On the other hand, the valence band of LaPTC $(+0.61 \mathrm{eV})[16]$ is less positive than HO$\mathrm{MO}$ of $\mathrm{MB}(+1.61 \mathrm{eV})$ and $\mathrm{RhB}(+1.10 \mathrm{eV})$ and oxidation potential $\left(\mathrm{H}_{2} \mathrm{O} / \mathrm{OH}\right)(+2.32 \mathrm{eV})$, therefore the photoexicet electron-hole pairs will be recombined and the formation of radical species does not occur and inhibit the degradation of methylene blue and rhodamin B [17]. Meanwhile, the HOMO of MO is less positive $(+0.14 \mathrm{eV})$ than the valence band of La-PTC and the LUMO of MO $(-1.87 \mathrm{eV})$ is more negative than the conduction band of La-PTC. The degradation of MO took place via the photosensitization degradation pathway [18]. The photon irradiated the system, MO molecule was photogenerated to the LUMO level, generating

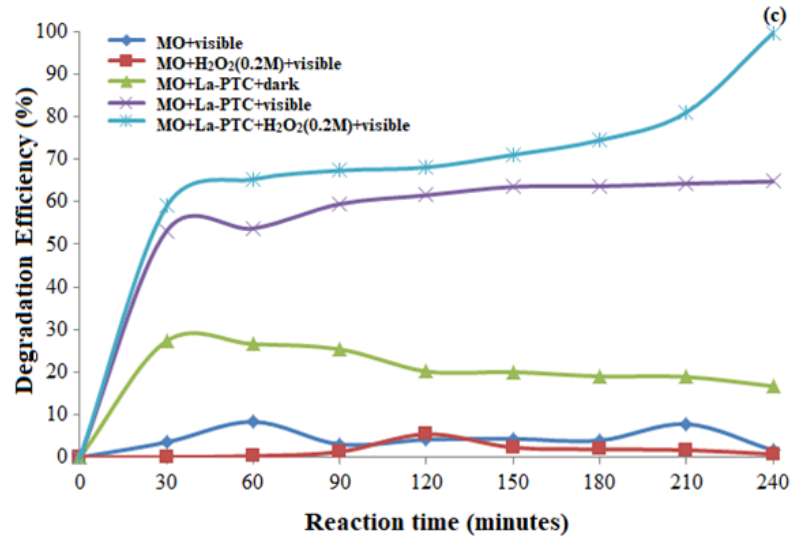

Figure 6. Percent degradation efficiency of (a) methylene blue (MB); (b) rhodamin B; and (c) methyl orange.
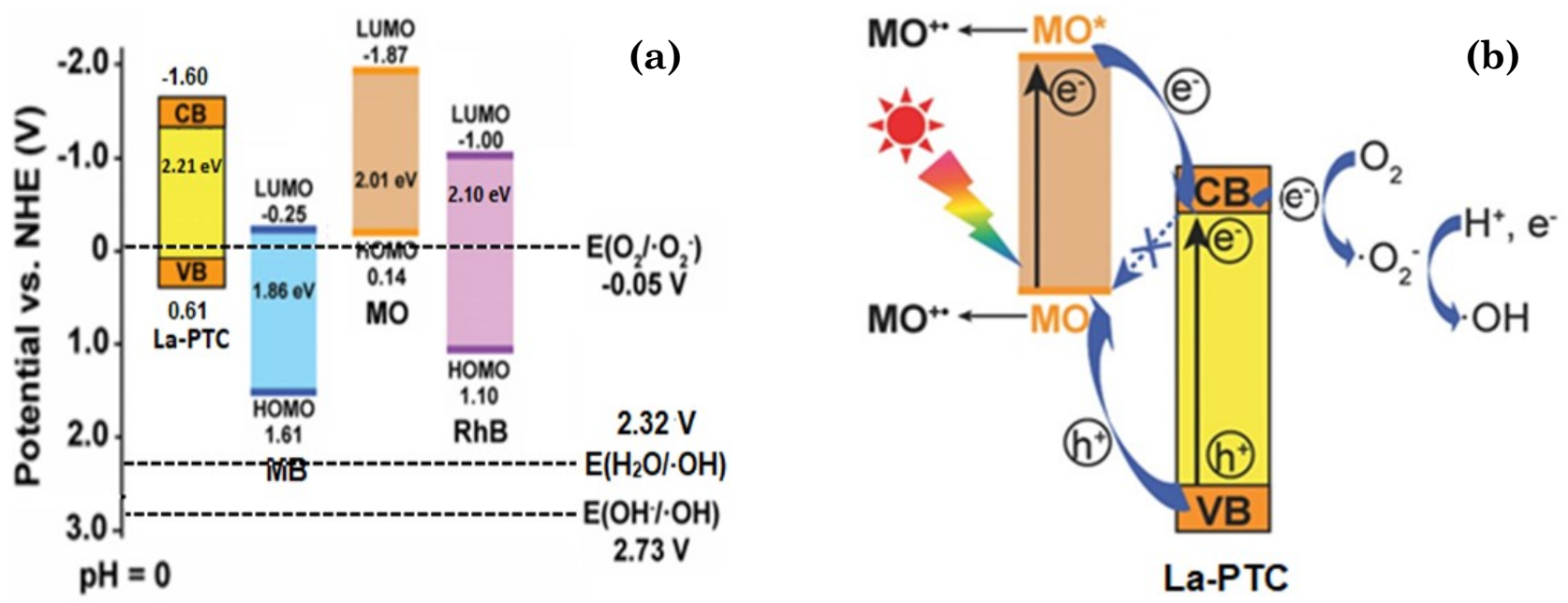

Figure 7. (a) The La-PTC band edge structures as well as HOMO and LUMO levels of MB, RhB, and MO vs. NHE at $\mathrm{pH}=0$, and (b) schematic illustration for MO degradation over La-PTC photocatalyst under visible-light irradiation. 
abundant of the photogenerated electrons at the LUMO level and holes at the HOMO level. These electrons can be an injection from the MO molecule to the conduction band (CB) of La-PTC, facilitating the generation of intermediate reactive species for starting dye degradation (Figure 7b).

Then, the photo-catalytic activity of MOF La-PTC was optimized by adjusting the amount of $\mathrm{H}_{2} \mathrm{O}_{2}$ for the $\mathrm{MB}, \mathrm{RhB}$ and $\mathrm{MO}$ degradation. As shown in Figure 6, the appearance of $\mathrm{H}_{2} \mathrm{O}_{2}$ elevated the dyes degradation efficiency by La-PTC about $67.02 \%$ for MB, $77.00 \%$ for $\mathrm{RhB}$ and $99.60 \%$ for MO. The replenishment of $\mathrm{H}_{2} \mathrm{O}_{2}$ would be an advantage for photocatalytic activity by generating a highly reactive intermediate of hydroxyl free radical [19]. In general, the photocatalytic activity of MOF La-PTC is associated with the electron charge transfer from the photoexcited organic ligand to the metal (LMCT) within MOFs accounts for their photocatalytic activity [20]. When La-PTC was irradiated with photons, the photoexcited electrons are transferred from the valence band (VB) to the conduction band (CB), accompanied by the formation of positive-charged holes $(\mathrm{h}+)$ in the VB [21]. $\mathrm{H}_{2} \mathrm{O}_{2}$ will react with photoexcited electrons to form $\cdot \mathrm{OH}$ radical and hinder photoexcited electron-hole pairs recombination. Simultaneously, electrons react with oxygen to form superoxide radicals $\left(\cdot \mathrm{O}_{2}^{-}\right)[22]$ and $\mathrm{h}^{+}$react with $\mathrm{H}_{2} \mathrm{O}$ or $\mathrm{OH}^{-}$form $\cdot \mathrm{OH}$ radical will also have a strong ability to oxidize dyes molecules.
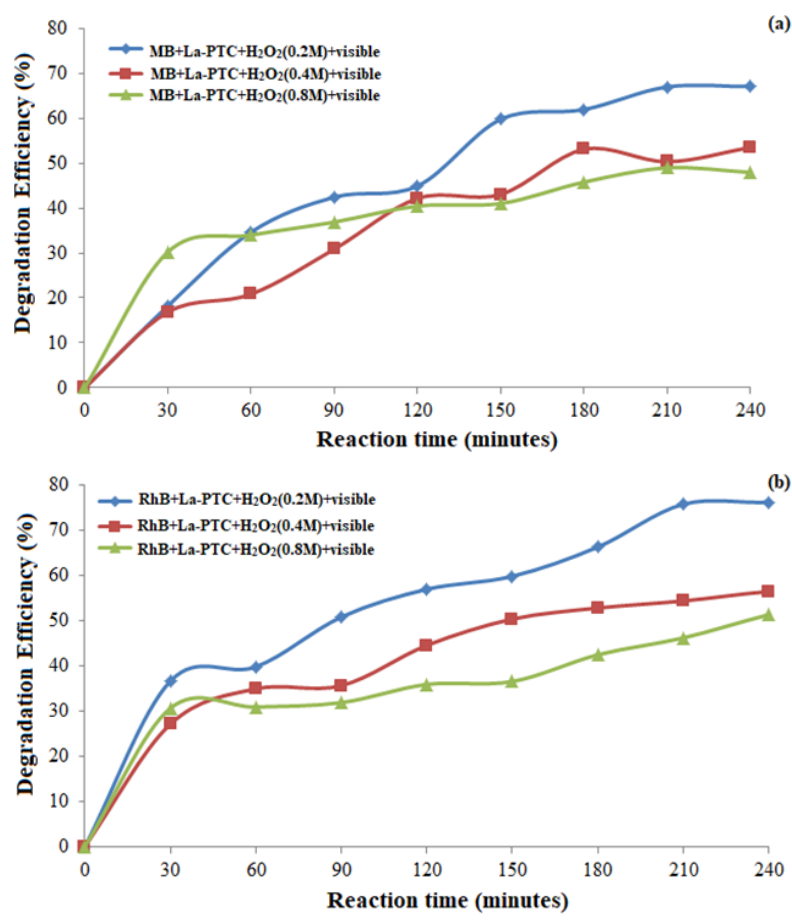

The influence of La-PTC and $\mathrm{H}_{2} \mathrm{O}_{2}$ concentration on $\mathrm{MB}$ and $\mathrm{MO}$ photocatalytic degradation then were evaluated [23]. As shown in Figure 8 , a furthermore increase of $\mathrm{H}_{2} \mathrm{O}_{2}$ induces a decrease of degradation of $\mathrm{MB}$ and $\mathrm{MO}$, suggesting the increasing $\mathrm{H}_{2} \mathrm{O}_{2}$ concentration destructed La-PTC active sites and decrease the photocatalytic performance. Abramovic and coworkers in 2015 reported that the additional excess of $\mathrm{H}_{2} \mathrm{O}_{2}$ as an electron scavenger will generate a hydroperoxyl $\left(\cdot \mathrm{HO}_{2}\right)$ radical then react strongly with hydroxyl radical $(\cdot \mathrm{OH})$ to form oxygen and water, causes the concentration of hydroxyl radical in the system was decreasing. Therefore, the subsequent photocatalytic $\mathrm{MB} / \mathrm{RhB} / \mathrm{MO}$ degradation was carried out in which $\mathrm{H}_{2} \mathrm{O}_{2} 0.2 \mathrm{M}$ was added [24].

To explain the main contributor species in the reaction, several studies verified the change of the degradation efficiency of MB/MO in the presence of different scavengers such as $\mathrm{H}_{2} \mathrm{O}_{2}$ as electron $\left(\mathrm{e}^{-}\right)$scavenger, methanol as hole $\left(\mathrm{h}^{+}\right)$scavenger and tert-butyl alcohol (TBA) as the $\mathrm{OH}$ scavenger [25].

Figure $8 \mathrm{a}$ and $8 \mathrm{~b}$ exhibit on the photodegradation of $\mathrm{MB}$ and $\mathrm{RhB}$, the activity increased in the presence of $\mathrm{H}_{2} \mathrm{O}_{2}$ and $\mathrm{CH}_{3} \mathrm{OH}$ than without the presence of $\mathrm{H}_{2} \mathrm{O}_{2}$ and $\mathrm{CH}_{3} \mathrm{OH}$. This happened because the addition of $\mathrm{H}_{2} \mathrm{O}_{2}$ and $\mathrm{CH}_{3} \mathrm{OH}$ can trigger the formation of the radical species, hence decreasing the rate of electron-hole recombination. When the TBA was adding in the system, the degradation of MB was slightly decrease due to the limited $\cdot \mathrm{OH}$ formed. Accordingly $\cdot \mathrm{OH}$ radical, $\cdot \mathrm{O}_{2}{ }^{-}$ radical, photo-excited electron and holes were the contributor in $\mathrm{MB}$ and $\mathrm{RhB}$ degradation with the major contributor were hole and $\cdot \mathrm{OH}$ [26]. Whilst the photo-degradation of MO was slightly enhanced with the addition of $\mathrm{H}_{2} \mathrm{O}_{2}$ and decreasing when the addition of $\mathrm{CH}_{3} \mathrm{OH}$ and TBA were applied (Figure 8c). This represented that the holes and $\cdot \mathrm{OH}$ radicals were major species that play a role in MO degradation.

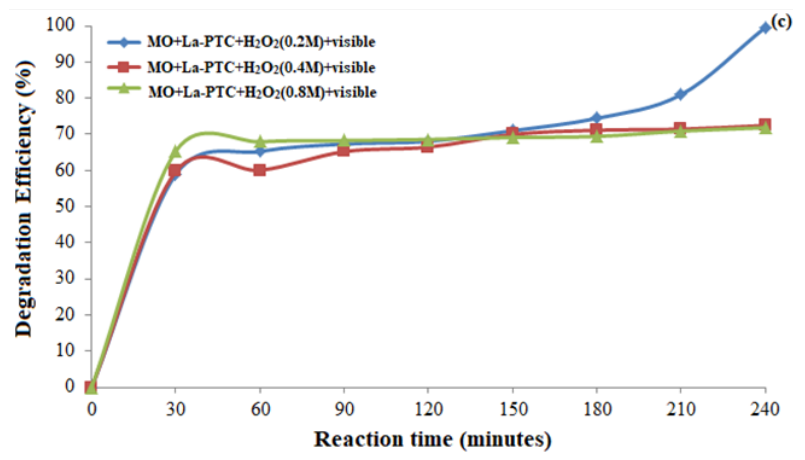

Figure 8. Degradation efficiency of (a) MB; (b). $\mathrm{RhB}$; and (c) $\mathrm{MO}$ in various $\mathrm{H}_{2} \mathrm{O}_{2}$ concentration. 
To assess the reusability of the La-PTC, recycling activities were carried out. La-PTC shows high photocatalytic activity for MB and $\mathrm{RhB}$ degradation during four cycle reactions with degradation efficiency of $77 \%$ for MB and $87 \%$ for $\mathrm{RhB}$, and MO degradation during three cycle reactions with degradation efficiency of $62.57 \%$ with the presence of $\mathrm{H}_{2} \mathrm{O}_{2}$ in the system.

\section{Conclusions}

The La-PTC MOF was synthesized by a solvothermal method using a perylene-3,4,9,10tetracarboxylate ligand and lanthanum metal ion. La-PTC has a broad-range visible light absorption with the main optical absorption band of La-PTC was around $561 \mathrm{~nm}$, high photocatalytic degradation activity of MO with the degradation efficiency of up to $99.60 \%$ in the presence of $\mathrm{H}_{2} \mathrm{O}_{2} . \mathrm{H}_{2} \mathrm{O}_{2}$-assisted $\mathrm{MB} / \mathrm{RhB} / \mathrm{MO}$ study suggested that adding the $\mathrm{H}_{2} \mathrm{O}_{2}$ into the system enhanced the photo-catalytic activity by quickly generating radical reactive species of hydroxyl. Hydroxyl species and holes are the contributor species in MO degradation. Meanwhile, electrons, superoxide radicals, hydroxyl radicals and holes are the contributor species in $\mathrm{MB}$ and $\mathrm{RhB}$ degradation, with hydroxyl radicals and holes as the main contributors.

\section{Acknowledgment}

The authors wish to thank the Ministry of Research and Technology/National Research and Innovation Agency of Indonesia (Kemenristek / BRIN) for financial support in carrying out this work through the research contract.

\section{References}

[1] Ramasundaram, S., Seid, M.G., Choe, J.W., Kim, E.-J., Chung, Y.C., Cho, K., Lee, S., Hong, S.W. (2016). Highly reusable $\mathrm{TiO}_{2}$ nanoparticle photocatalyst by direct immobilization on steel mesh via PVDF coating, electrospraying, and thermal fixation. Chemical Engineering Journal, 306, 344-351, doi: 10.1016/j.cej.2016.07.077.

[2] Hoffmann, M.R., Martin, S.T., Choi, W., Bahnemann, D.W. (1995). Environmental Applications of Semiconductor Photocatalysis. Chemical Reviews, 1(95), 69-96, doi: 10.1021/cr00033a004.

[3] Zou, X., Zhang, Y. (2015). Noble metal-free hydrogen evolution catalysts for water splitting. Chemical Society Reviews, 44(15), 51485180, doi: 10.1039/c4cs00448e.
[4] Lu, W., Wei, Z., Gu, Z.-Y., Liu, T.-F., Park, J., Park, J., Tian, J., Zhang, M., Zhang, Q., Gentle III, T., Bosch, M., Zhoum, H.-C. (2014). Tuning the structure and function of metalorganic frameworks via linker design. Chemical Society Reviews, 43(16), 5561-5593, doi: 10.1039/c4cs00003j.

[5] Rojas, S., Horcajada, P. (2020). Metal - Organic Frameworks for the Removal of Emerging Organic Contaminants in Water. Chemical Reviews, 120, 16, 8378-8415, doi: 10.1021/acs.chemrev.9b00797.

[6] Wang, C.C., Li, J.R., Lv, X.L., Zhang, Y.Q., Guo, G. (2014). Photocatalytic organic pollutants degradation in metal-organic frameworks. Energy \& Environmental Science, 7(9), 2831-2867, doi: 10.1039/c4ee01299b.

[7] Yuan, S., Feng, L., Wang, K., Pang, J., Bosch, M., Lollar, C., Sun, Y., Qin, J., Yang, X., Zhang, P., Wang, Q., Zou, L., Zhang, Y., Zhang, L., Fang, Y., Li, J., Zhou, H.-C. (2018). Stable Metal-Organic Frameworks: Design, Synthesis, and Applications. Advanced Materials, $\quad 30(37), \quad 1704303$, doi: 10.1002/adma.201704303.

[8] Xia, Q., Yu, X., Zhao, H., Wang, S., Wang, H., Guo, Z., Hongzhu Xing, H. (2017). Syntheses of Novel Lanthanide Metal-Organic Frameworks for Highly Efficient Visible-LightDriven Dye Degradation. Crystal Growth \& Design, 17(8), 4189-4195, doi: 10.1021/acs.cgd.7b00504.

[9] Buhori, A., Zulys, A., Gunlazuardi, J. (2020). Synthesis of Lanthanum metal-organic frameworks (La-MOFs) as degradation photocatalyst of Rhodamine-B. AIP Conference Proceedings, 2242, 040033, doi: 10.1063/5.0013010.

[10] Pan, W., Gong, C., Zeng, X., Hu, C., Zhang, Y., Zhu, D.-R., Xu, H., Guo, H., Zhang, J., Xie, J. (2019). Assembly of porous lanthanide metal-organic frameworks constructed by chalcone dicarboxylic acid and exploration of their properties. Polyhedron, 169, 24-31, doi: 10.1016/j.poly.2019.04.062.

[11] Batubara, N.H., Zulys, A. (2019). Synthesis, Structural, Spectroscopic, and Morphology of Metal-Organic Frameworks Based on la (III) and Ligand 2,6-Napthalenedicarboxylic acid (La-MOFs) for Hydrogen Production. IOP Conference Series: Materials Science and Engineering, 546(4), 042005, doi: 10.1088/1757899X/546/4/042005.

[12] Ambroz, F., Macdonald, T.J., Martis, V., Parkin, I.P. (2018). Evaluation of the BET Theory for the Characterization of Meso and Microporous MOFs. Small Methods, 2(11), 1800173, doi: 10.1002/smtd.201800173. 
[13] Christina, L.C., Gunlazuardi, J., Zulys, A. (2020). Synthesis and characterization of lanthanide metal-organic framework with perylene 3,4,9,10-tetracarboxylate ligand. IOP Conference Series: Materials Science and Engineering, 902(1), 012046, doi: 10.1088/1757-899X/902/1/012046.

[14] Zulys, A., Asrianti, D., Gunlazuardi, J. (2020). Synthesis and characterization of metal organic frameworks based on nickel and perylene dyes as water splitting photocatalyst. AIP Conference Proceedings, 2243, 020035, doi: 10.1063/5.0005001.

[15] Chang, S.-S., Clair, B., Ruelle, J., Beauchêne, J., Di Renzo, F., Quignard, F., Zhao, G.-J., Yamamoto, H., Gril, J. (2009). Mesoporosity as a new parameter for understanding tension stress generation in trees. Journal of $E x$ perimental Botany, 60(11), 3023-3030, doi: 10.1093/jxb/erp133.

[16] Zulys, A., A’Yun, Q., Gunlazuard, J. (2020). Synthesis of metal organic frameworks based on lanthanum metal and perylene ligand as photocatalyst for hydrogen gas production. AIP Conference Proceedings, 2243, 020034, doi: 10.1063/5.0005000.

[17] Kudo, A., Miseki, Y. (2009). Heterogeneous photocatalyst materials for water splitting. Chemical Society Reviews, 38(1), 253-278, doi: 10.1039/b800489g.

[18] Pingmuang, K., Chen, J., Kangwansupamonkon, W., Wallace, G.G., Phanichphant, S., Nattestad, A. (2017). Composite Photocatalysts Containing $\mathrm{BiVO}_{4}$ for Degradation of Cationic Dyes. Scientific Reports, 7(1), 8929, doi: 10.1038/s41598-017-09514-5.

[19] Li, N., Xu, J., Feng, R., Hu, T.L., Bu, X.H. (2016). Governing metal-organic frameworks towards high stability. Chemical Communications, 52(55), 8501-8513, doi: $10.1039 / \mathrm{c} 6 \mathrm{cc} 02931 \mathrm{k}$.
[20] Chong, M.N., Jin, B., Chow, C.W.K., Saint, C. (2010). Recent developments in photocatalytic water treatment technology: A review. Water Research, 44(10), 2997-3027, doi: 10.1016/j.watres.2010.02.039.

[21] Al-mamun, M.R., Kader, S., Islam, M.S., Khan, M.Z.H. (2019). Photocatalytic activity improvement and application of $\mathrm{UV}_{-} \mathrm{TiO}_{2}$ photocatalysis in textile wastewater treatment: A review. Journal of Environmental Chemical Engineering, 7(5), 103248, doi: 10.1016/j.jece.2019.103248.

[22] Isac, L., Cazan, C., Enesca, A., Andronic, L. (2019). Copper Sulfide Based Heterojunctions as Photocatalysts for Dyes Photodegradation. Frontiers in Chemistry, 7, 694, doi: 10.3389/fchem.2019.00694.

[23] Natarajan, S., Bajaj, H.C., Tayade, R.J. (2018). Recent advances based on the synergetic effect of adsorption for removal of dyes from waste water using photocatalytic process. Journal of Environmental Sciences, 65, 201-222, doi: 10.1016/j.jes.2017.03.011.

[24] Abramovic, B., Despotovic, V., Šojic, D., Fincur, N. (2015). Mechanism of clomazone photocatalytic degradation: Hydroxyl radical, electron and hole scavengers. Reaction Kinetics, Mechanisms and Catalysis, 115(1), 67-79, doi: 10.1007/s11144-014-0814-z.

[25] Pu, Y.C., Chou, H.Y., Kuo, W.S., Wei, K.H., Hsu, Y.J. (2017). Interfacial charge carrier dynamics of cuprous oxide-reduced graphene oxide $\left(\mathrm{Cu}_{2} \mathrm{O}\right.$-rGO) nanoheterostructures and their related visible-light-driven photocatalysis. Applied Catalysis B: Environmental, 204, 21-32, doi: 10.1016/j.apcatb.2016.11.012.

[26] Chiu, Y.H., Chang, T.F.M., Chen, C.Y., Sone, M., Hsu, Y.J. (2019). Mechanistic insights into photodegradation of organic dyes using heterostructure photocatalysts. Catalysts, 9(5), 430, doi: 10.3390/catal9050430.

Selected and Revised Papers from $3^{\text {rd }}$ International Conference on Chemistry, Chemical Process and Engineering 2020 (IC3PE 2020) (https://chemistry.uii.ac.id/ic3pe/) (Universitas Islam Indonesia (UII), Labuan Bajo, Nusa Tenggara Timur, Indonesia by 30th September - 1st October 2020) after Peer-reviewed by Scientific Committee of IC3PE 2020 and Peer-Reviewers of Bulletin of Chemical Reaction Engineering \& Catalysis. Editors: I. Istadi; Is Fatimah 